\title{
Antimalarial therapy prevents Myc-induced lymphoma
}

\author{
Chi V. Dang
}

Division of Hematology, Department of Medicine, Johns Hopkins University School of Medicine, Baltimore, Maryland, USA.

\begin{abstract}
The two modes of self-destruction at the cellular level - apoptosis (selfkilling) and autophagy (self-eating) - are thought to be tumor suppressive. In particular, germline loss of function of genes involved in autophagy has been associated with tumorigenesis. However, recent studies, including the one by Maclean et al. reported in this issue of the JCI, indicate that autophagy can provide a means for cell survival when nutrients are limiting, such that inhibition of autophagy by the antimalarial drug chloroquine can inhibit tumorigenesis, specifically $M y c$-induced lymphoma in mice (see the related article beginning on page 79 ). These findings suggest that a new use of an old drug for cancer prevention may profoundly affect disease outcome.
\end{abstract}

Burkitt lymphoma occurs endemically in Africa, sporadically elsewhere, and in association with HIV/AIDS worldwide. Endemic Burkitt lymphoma, a high-proliferativeindex (high-growth-rate) B cell malignancy initially described in 1958 by Denis Burkitt, an Irish surgeon working in Uganda, has been epidemiologically associated with the Epstein-Barr virus and Plasmodium falciparum malaria in Africa (1). These links suggest that Burkitt lymphoma is a polymicrobial B lymphocytic disease triggered by microbial-mediated immune stimulation and acquisition of spontaneous oncogenic mutations due to the ability of $B$ cells to generate antibody diversity through DNA recombination. In this regard, chloroquine prophylaxis against malaria in Tanzania, reported in 1987, was associated with a reduction in the number of cases of Burkitt lymphoma (2). This lymphoma was one of the first human cancers for which disease pathogenesis was linked to the activation of an oncogene, in this case c-MYC (also known as MYC) (3). Although there were questions regarding the cause and effect of malaria prophylaxis and the associated reduction of lymphoma in Africa at that time, the report by Maclean and coworkers in this issue of the $J C I$ demonstrates that chloroquine can prevent Burkitt-like lymphoma in a transgenic

Nonstandard abbreviations used: ATG4, autophagyrelated protein 4.

Conflict of interest: The author has declared that no conflict of interest exists.

Citation for this article: J. Clin. Invest. 118:15-17 (2008). doi:10.1172/JCI34503. mouse model (4). However, instead of chloroquine being shown to modulate malaria, the current work of Maclean et al. and previous work from Thompson and coworkers demonstrate that the antilymphoma effects of chloroquine are mediated through suppression of lysosome-mediated degradation of damaged proteins or organelles - a process known as "autophagy" (4-6).

\section{Oncogenic MYC drives metabolic needs}

Normal cells, as compared with cancer cells, have checks and balances that diminish proliferation and attenuate cellular metabolism when nutrients or oxygen are limiting $(6,7)$. In this regard, normal cells withdraw from the cell cycle and decrease oxidative phosphorylation when there is inadequate oxygen (hypoxia) and nutrients $(7,8)$. Unlike normal cells, cancer cells have oncogenic mutations that drive cellular growth (cell size increase) and proliferation (Figure 1), even in the face of limited oxygen and nutrients. These limitations are frequently found in the tumor microenvironment, which is characterized by inadequate and abnormal neovasculature (6). Activation of the MYC oncogene, which encodes the MYC transcription factor, results in cellular growth through MYC activation of genes involved in ribosomal biogenesis and energy metabolism $(3,9)$. Together with enforced expression of genes involved in cell cycle progression, MYC induces abnormal cell proliferation (Figure 1). The proliferative drive by Myc increases the transformed cell's energy demand such that nutrient limitation triggers p53- or ARF-dependent apoptosis (10). Tumor cells can escape this fate of death by either activating the pathway of self-ingestion (autophagy) to garner sufficient energy to survive through a period of nutrient limitation or by eliminating p53 or ARF via mutations (Figure 1) $(6,10)$.

\section{Autophagy as a means for survival}

Distinct from apoptosis, autophagy can be envisioned to be another mode of selfdestruction whereby the cell eats itself to death (Figure 1) $(11,12)$. However, the activation of autophagy following nutrient deprivation or hypoxia suggests that autophagy plays a homeostatic role in response to oxidative stress triggered by these same conditions (12). Hence, autophagy degrades damaged organelles and recycles them as an alternative energy source in response to prolonged metabolic stress (13). When cells are deprived of nutrients in the presence of a trophic growth factor signal, apoptosis is activated unless the apoptotic machinery is disabled. For example, deletion of Bax and Bak, the pro-death BH3-domain mitochondrial membrane proteins, inhibits apoptosis in response to nutrient deprivation (6). The lack of extracellular nutrients forces the cell to derive its energy from internal sources through autophagy. Thus inhibition of autophagy results in cell death by provoking bioenergetic failure that triggers necrosis, rather than preventing the cell from eating itself to death. It should be noted however, that there is only so much that a cell can consume of itself. Hence, autophagy in the absence of apoptosis under nutrient deprivation would ultimately culminate in the death of a cell.

There are various mechanisms that activate autophagy. In the context of a tumor cell, hypoxia and nutrient deprivation both increase ROS generation by the mitochondria. In turn, ROS are known to increase levels of hypoxia-inducible factor 1 (HIF-1) by inactivating prolyl hydroxylases, which modify HIF- $1 \alpha$ for proteasomal degradation (7). HIF-1, a dimer of HIF- $1 \alpha$ and HIF-1 $\beta$, 


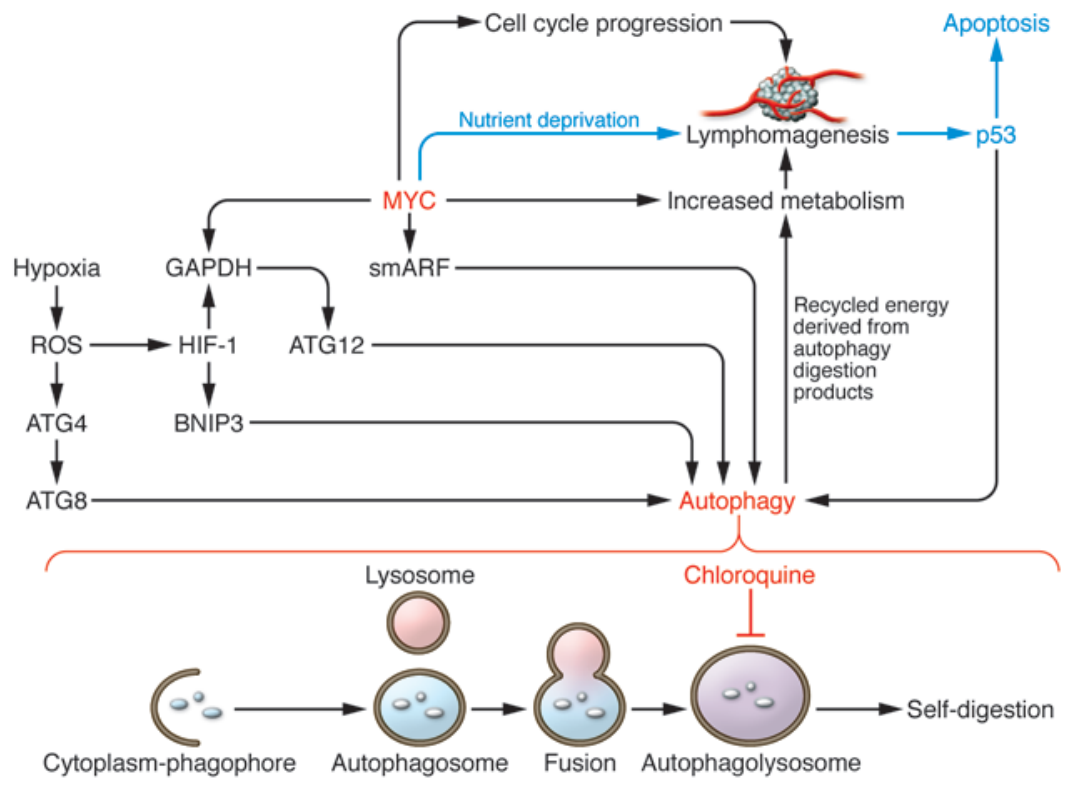

Figure 1

Inhibition of autophagy by chloroquine blocks recycling of energy and MYC-mediated lymphomagenesis. Activated MYC induces lymphomagenesis (depicted as a cluster of cells with neovasculature) via activation of genes involved in cell cycle progression, which increases metabolic demand. This demand is in part met by the upregulation of MYC target genes involved in energy metabolism. MYC also induces p53, which triggers apoptosis in the setting of nutrient deprivation, unless the autophagy pathway can be activated to fulfill the energetic needs of the MYC-transformed cancer cell. MYC is depicted to activate a small mitochondrial isoform of ARF known as "smARF," which translocates into mitochondria, triggering autophagy. Hypoxia is depicted to increase ROS, which in turn increase the levels of HIF-1. HIF-1 induces BNIP3 and, with MYC, can induce GAPDH. Both BNIP3 and GAPDH have been shown to regulate autophagy $(14,17)$. In addition, ROS modulate ATG4, permitting induction of autophagy via ATG8 (19). Autophagy occurs through assembly of cytoplasmic components within a membranous phagophore, which results in the formation of an autophagosome. A lysosome fuses with an autophagosome to form an autophagolysosome. In the report by Maclean and coworkers in this issue of the $\mathrm{JCl}$, chloroquine is demonstrated to inhibit the final step in the autophagy pathway - the degradation/digestion of cargo within the autophagolysosome in order to provide recycled energy for the cell — thereby preventing MYC-mediated lymphomagenesis (4).

transactivates BNIP3, which is a BH3domain mitochondrial protein known to induce autophagy through a mechanism that remains to be fully delineated (Figure 1) $(8,14)$. ROS also modifies autophagy-related protein 4 (ATG4), triggering autophagy and mitophagy (the autoingestion of damaged mitochondria) (Figure 1) $(15,16)$. Intriguingly, other target genes of MYC and HIF have also been recently implicated in autophagy. HIF and MYC both activate GAPDH, whose role as a glycolytic enzyme and as a transcriptional cofactor stimulates autophagy (Figure 1) (17). Furthermore, MYC induction of the tumor suppressor $\mathrm{p} 19^{\mathrm{ARF}}$ produces both the full-length ARF and a smaller mitochondrial isoform termed "smARF," which induces autophagy (Figure 1) (18). Hence, in a hypoxic MYC-induced tumor cell, which has a high metabolic demand, autophagy is a strategy for sustaining the tumor cell's energy needs such that full inhibition of autophagy is expected to induce cell death and diminish tumorigenesis.

When nutrients are available to cells, target of rapamycin (TOR), a key sensor of a cell's nutrient status, phosphorylates and inhibits the autophagy-promoting proteins ATG1 and ATG13, thereby disabling autophagy (19). Under conditions of nutrient deprivation, activated ATG1 and ATG13 are dephosphorylated and form a complex that triggers the formation of autophagosomes, which ultimately fuse with lysosomes to form autophagolysosomes for self-digestion (Figure 1) (12). The products of digestion are recycled as energy and building blocks for continued cell survival during a period of starvation. Maclean and coworkers have shown that chloroquine does not block the docking and fusion of autophagosomes with lysosomes as previously thought, but rather inhibits the degradation of cargo delivered to the lysosome, thus effectively disabling this final step of the autophagy pathway (Figure 1). Indeed, Amaravadi and coworkers previously reported in the JCI that inhibition of autophagy by chloroquine increases therapy-induced cell death in a Myc-induced model of murine lymphoma (5). They also demonstrated a dependence on p53 in this model.

\section{Use of an old drug for a new indication?}

The report by Maclean and coworkers in this issue of the JCI provides evidence that chloroquine could be used for cancer chemoprevention, at least in transgenic mouse models of lymphomas induced by Myc or Atm deficiency (4). This team reasoned that since chloroquine activates the Atm-p53 stress-responsive pathway, it could induce cell death. Indeed chloroquine was found to induce cell death in a p53-dependent manner, but surprisingly this effect was independent of Atm or Arf. In this regard, chloroquine inhibited lymphomagenesis in mice with Atm-deficient lymphomas, but it could not prevent lymphomas that arose in p53-deficient mice. Furthermore, the authors studied the transgenic pretumor Myc-overexpressing lymphocytes ex vivo and observed that chloroquine induced cell death by impairing autophagic lysosomal protein degradation that could not be rescued by Bcl-2 or Bcl- $\mathrm{X}_{\mathrm{L}}$ (Figure 1 ). These observations provide additional preclinical evidence that chloroquine could be effective as a chemoprevention agent, partic- 
ularly since the results of the study by Geser et al. suggest that malarial prophylaxis with chloroquine diminished the incidence of Burkitt lymphomas in Tanzania (2). In this regard, antioxidants, which have also been implicated in blocking autophagy, also prevent tumor formation in transgenic mice and xenograft models $(16,20)$. With chloroquine, this antimicrobial agent was initially thought to diminish lymphomagenesis by inhibiting malaria, but in fact its ability to inhibit autophagy through blocking lysosomalmediated degradation as demonstrated by the current study by Maclean et al. appears to underpin its antitumorigenic activity in the clinical epidemiological setting (4). These instructive, insightful observations suggest that the use of chloroquine or its improved versions may prove to have a major impact in cancer prevention. It is notable, however, that the long-term effects of prolonged use of a potent autophagy inhibitor may have unexpected side effects, as our understanding of the homeostatic role of autophagy in normal tissues is rudimentary.

\section{Acknowledgments}

I apologize for omission of primary references and have largely relied on review articles for this commentary. I thank J. Cleveland, P. Gao, M. Kastan, J. Kim, and J. Yustein for their input.

Address correspondence to: Chi V. Dang, Johns Hopkins University School of Medicine, Ross Research Building, Room 1032, 720 Rutland Avenue, Baltimore, Maryland 21205, USA. Phone: (410) 955-2411; Fax: (410) 955-0185; E-mail: cvdang@jhmi.edu.

1. van den Bosch, C.A. 2004. Is endemic Burkitt's lymphoma an alliance between three infections and a tumour promoter? Lancet Oncol. 5:738-746.

2. Geser, A., Brubaker, G., and Draper, C.C. 1989. Effect of a malaria suppression program on the incidence of African Burkitt's lymphoma. Am.J. Epidemiol. 129:740-752.

3. Dang, C.V., O’Donnell, K.A., Zeller, K.I., Nguyen, T., Osthus, R.C., and Li, F. 2006. The c-Myc target gene network. Semin. Cancer Biol. 16:253-264.

4. Maclean, K.H., Dorsey, F.C., Cleveland, J.L., and Kastan, M.B. 2008. Targeting lysosomal degradation induces p53-dependent cell death and prevents cancer in mouse models of lymphomagenesis. J. Clin. Invest. 118:79-88.

5. Amaravadi, R.K., et al. 2007. Autophagy inhibition enhances therapy-induced apoptosis in a Myc-induced model of lymphoma. J. Clin. Invest. 117:326-336

6. Lum, J.J., et al. 2005. Growth factor regulation of autophagy and cell survival in the absence of apoptosis. Cell. 120:237-248.

7. Brahimi-Horn, M.C., Chiche, J., and Pouyssegur, J. 2007. Hypoxia signalling controls metabolic demand. Curr. Opin. Cell Biol. 19:223-229.

8. Semenza, G.L. 2007. Hypoxia-inducible factor 1 (HIF-1) pathway. Sci. STKE. 2007:cm8.

9. Zeller, K.I., et al. 2006. Global mapping of c-Myc binding sites and target gene networks in human B cells. Proc. Natl. Acad. Sci. U. S. A. 103:17834-17839.

10. Cleveland, J.L., and Sherr, C.J. 2004. Antagonism of Myc functions by Arf. Cancer Cell. 6:309-311.

11. Rubinsztein, D.C., Gestwicki,J.E., Murphy, L.O., and Klionsky, D.J. 2007. Potential therapeutic applications of autophagy. Nat. Rev. Drug Discov. 6:304-312.

12. Maiuri, M.C., Zalckvar, E., Kimchi, A., and Kroemer, G. 2007. Self-eating and self-killing: crosstalk between autophagy and apoptosis. Nat. Rev. Mol. Cell Biol. 8:741-752.

13. Jin, S., and White, E. 2007. Role of autophagy in cancer: management of metabolic stress. Autophagy. 3:28-31.

14. Tracy, K., et al. 2007. BNIP3 is an RB/E2F target gene required for hypoxia-induced autophagy. Mol. Cell. Biol. 27:6229-6242.

15. Scherz-Shouval, R., et al. 2007. Reactive oxygen species are essential for autophagy and specifically regulate the activity of Atg4. EMBO J. 26:1749-1760.

16. Scherz-Shouval, R., and Elazar, Z. 2007. ROS, mitochondria and the regulation of autophagy. Trends Cell Biol. 17:422-427.

17. Colell, A., et al. 2007. GAPDH and autophagy preserve survival after apoptotic cytochrome c release in the absence of caspase activation. Cell. 129:983-997.

18. Reef, S., et al. 2006. A short mitochondrial form of p19ARF induces autophagy and caspase-independent cell death. Mol. Cell. 22:463-475.

19. Kamada, Y., et al. 2000. Tor-mediated induction of autophagy via an Apg1 protein kinase complex. J. Cell Biol. 150:1507-1513.

20. Gao, P., et al. 2007. HIF-dependent antitumorigenic effect of antioxidants in vivo. Cancer Cell. 12:230-238.

\title{
Hypertensive encephalopathy and the blood-brain barrier: is $\delta P K C$ a gatekeeper?
}

\author{
Wen-Hai Chou and Robert O. Messing
}

Ernest Gallo Clinic and Research Center, Department of Neurology, UCSF, Emeryville, California, USA.

\begin{abstract}
Hypertensive encephalopathy is a life-threatening condition due to elevation of cerebral perfusion pressure beyond the limits of autoregulation. Breakdown of the blood-brain barrier (BBB) leads to cerebral edema and reduced blood flow. In this issue of the JCI, Mochly-Rosen and colleagues demonstrate a novel molecular strategy for preserving the BBB in a model of hypertension-induced encephalopathy (see the related article beginning on page 173). Using a rationally designed peptide inhibitor of $\delta P K C$, they stabilized the BBB and improved mortality in hypertensive rats. This study highlights the therapeutic potential of $\delta$ PKC inhibitors in hypertensive encephalopathy and provides incentive to elucidate $\delta \mathrm{PKC}$ signaling pathways that mediate $\mathrm{BBB}$ dysfunction in other disease states.
\end{abstract}

Nonstandard abbreviations used: as-, analog sensitive; BBB, blood-brain barrier; RACK, receptor for $\underline{\text { activated }} \underline{\mathrm{c}}$-kinase; TAT, transactivator of transcription; ZO-1, zonula occludens 1.

Conflict of interest: The authors have declared that no conflict of interest exists.

Citation for this article: $J$. Clin. Invest. 118:17-20 (2008). doi:10.1172/JCI34516.

\section{The blood-brain barrier}

The blood-brain barrier (BBB) is a gliovascular unit composed of capillary endothelial cells and pericytes surrounded by basal lamina, astrocytic end-feet, and perivascular interneurons (Figure 1A) (1). The tight junctions formed between endothelial cells act as a highly effective physical barrier to selectively permit the entry of required nutrients yet protect the central nervous system from pathogens and potentially harmful small molecules circulating in the blood. The tight junction consists of transmembrane proteins (claudin, occludin, and junction adhesion molecule) and cytoplasmic accessory proteins (Figure 1B) (2). Claudins form dimers and bind to claudins on adjacent endothelial cells to establish the primary gate of the tight junction. The main functions of occludin appear to be to regulate the electrical resistance across the barrier and decrease paracellular permeability. Several accessory cytoplasmic proteins associate with these transmembrane components. Zonula occludens proteins ( $\mathrm{ZO}-1, \mathrm{ZO}-2$, and $\mathrm{ZO}-3)$ serve as recognition proteins for 